\title{
Voice source model for continuous control of pitch period
}

\author{
Paul H. Milenkovic \\ Department of Electrical and Computer Engineering, University of Wisconsin-Madison, 1415 Johnson Drive. \\ Madison, Wisconsin 53706
}

(Received 6 January 1992; revised 19 October 1992; accepted 23 October 1992)

\begin{abstract}
The voiced speech waveform may be synthesized by exciting an LPC vocal tract filter with a pulse waveform patterned after naturally occurring glottal airflow pulses. Such a pulse waveform may be generated by computing samples of a piecewise polynomial curve at equally spaced time intervals. In this type of synthesis, the pitch period is commonly restricted to an integer multiple of the sample interval. A method is presented for removing this restriction, permitting both pulse duration and pitch period to be varied over continuous time. Aliasing distortion is prevented by computing the sample values of pulses that have been low-pass filtered in continuous time prior to sampling. Applications of this technique include modeling glottal pulses by least-squares fit to inverse filter waveforms, the synthesis of calibration waveforms for evaluating measures of speech waveform jitter, the perceptual evaluation of low levels of waveform jitter, and the synthesis of the singing voice.
\end{abstract}

PACS numbers: 43.72.Ar, 43.72.Ja

\section{INTRODUCTION}

Voiced speech may be synthesized by applying excitation pulses to a digital filter according to the source-filter model of speech production. The natural glottal pulse contributes zeros to the speech spectrum (Mathews et al., 1961) which are understood to have perceptual relevance. Patterning the shape of the excitation pulses after the glottal pulses observed by inverse filtering was reported by Rosenberg (1971) and by Holmes (1973) to improve the naturalness of synthetic speech.

We have reported (Milenkovic, 1986) on the use of pulses described by piecewise polynomial curves to model the voice source. The voice source signal in this instance refers to the first derivative of glottal airflow as obtained by inverse filtering the speech waveform measured with a pressure microphone; the model pulses were patterned after the shape of the first derivative of the glottal airflow pulse. The parameters controlling the pulse shape were adjusted to fit the model to the inverse filtered speech waveform in a leastsquares sense.

Fully natural sounding speech synthesis may need to account for the effects of source-tract interaction. The vocal tract driving point impedance has a transform zero at dc related to the inertia of the air column, and this impedance zero accounts for the skewing of the glottal airflow pulse relative to a more symmetric glottal opening area pulse (Rothenberg, 1983). The vocal tract impedance also has peaks at the formant frequencies; these peaks result in formant ripple being added to the glottal airflow pulse (Ananthapadmanabha and'Fant, 1982).

Recent examples of source interactive synthesizers are reported by Sondhi and Schroeter (1988) and by Pinto et al. (1989). The Sondhi-Schroeter synthesizer is based on an articulatory model that produces a vocal tract area function from which both the vocal tract transfer function as well as the driving point impedance may be computed. The synthesizer described by Pinto et al. is based on a formant model where the formant frequencies are used to specify an equivalent circuit model of the driving point impedance.

Source interactive speech synthesis is restricted in its application by the need for articulatory information. The formant frequencies do not specify a unique vocal tract area function (Atal et al., 1978), and the different area functions with the same formant frequencies produce nearly similar transfer functions but widely different driving point impedances (Milenkovic, 1984). While the impedance peak frequencies are coincident with the formant frequencies, the impedance zero frequencies are not set by the formant frequencies, and the location of these zeros has a profound effect on the amplitudes of the impedance peaks affecting the formant ripple.

The effect of source-tract interaction on the acoustic waveform can, to a degree, be reproduced by a source-filter synthesizer. The skewing of the glottal airflow pulse is easily incorporated into a mathematical function describing a pulse shape. Formant ripple, however, is related to the dissipation of the formant oscillations into losses in the glottal constriction and subglottal acoustic system (Flanagan, 1972). Klatt and Klatt (1990) suggest using a pitch synchronous adjustment of bandwidths in a formant synthesizer to approximate this effect. On the other hand, the LPC multipulse synthesis method (Atal and Remde, 1982) is able to match the acoustic speech waveform in a least-squares sense using an LPC vocal tract filter that is fixed over one or more pitch period cycles. A model combining an LPC derived filter with a small number of source parameters may have sufficient degrees of freedom to approximate acoustic waveforms influenced by source-tract interaction.

Efforts to improve the quality of source-filter synthesized speech remain a worthwhile endeavor. In light of work in source-interactive synthesizers, the source-filter model is 
still employed to study the influence of the voice source on synthesis quality (Childers and Lee, 1991). While the source-filter synthesizer may never be as parsimonious as a source interactive synthesizer, the parameters controlling a source-filter synthesizer may be more readily determined by analysis of the acoustic speech waveform in the absence of $x$ ray measurements of vocal tract geometry. While the sourcefilter synthesizer may never give a perfect reproduction of natural speech waveforms, we seek to remedy sources of artifact where possible.

The process of fitting pulses to the voice source in discrete time is a source of artifact we seek to remedy. Fitting synthesizer pulses to the inverse filter waveform takes place in discrete time because that is the form in which we acquire speech waveforms for analysis. Because of discontinuous derivatives at the points of glottal opening and glottal closure, the synthesizer pulses will undergo aliasing distortion upon computing sample values. This aliasing distortion becomes evident when the pulse end points are not aligned with the sample points. The end points of the naturally occuring glottal pulses, however, are not necessarily aligned with the sample points.

We have modeled a glottal pulse with an end point lying between two sample points as a weighted average of pulses with end points placed at sample points on either side of the desired endpoint (Milenkovic, 1986). The pulses in the model need to be low-pass filtered well below the Nyquist frequency to make this averaging of pulses bracketing the desired end point a reasonable approximation to a pulse with the desired end point. This scheme gave good least-squares fits between synthetic and natural glottal pulses. The pitch period estimates obtained by using the pulse weights to interpolate between sample points gave values for jitter well below the sample interval, jitter being the cycle-to-cycle fluctuation in the pitch period. While the resynthesized speech waveform matched the large scale oscillations of the dominant formants in the natural speech waveform, the high frequencies were missing on account of the low-pass filter used to interpolate pulse end points.

We focus attention on devising a glottal pulse model of controllable bandwidth and spectrum slope that can be aligned between sample points. One solution would be to oversample the synthesizer pulse shape, digitally low-pass filter, and then resample at the rate of the digital speech recording as reported by Hartwell and Prezas (1981). The pulse end points would still be aligned on sample intervals, but the sample intervals would be much shorter. Another solution would be to put fillets or smooth corners on the pulse end points. This method was demonstrated by Titze (1986) to improve the naturalness of a synthesized singing voice. Of course the smooth corners significantly modify the voice source spectrum as well as reduce the aliasing artifact.

We have developed a technique for analytically applying a low-pass antialias filter to pulses described by piecewise polynomial curves. The filter is effectively applied in continuous time before the sample valuies are obtained. We are able to compute sample values of polynomial pulse that is lowpass filtered close to the Nyquist frequency, allowing continuous control of pulse duration and pitch period with mini- mal levels of aliasing distortion. We also have control over the spectral shape of the antialias filtering.

While the derivation of the technique may seem complicated, the actual computation of these pulse shapes is quite straightforward. Sample values are obtained by multiplying vectors containing powers of time $t$, sine, and cosine values by matrices of constant coefficients. The computations compare quite favorably with computing pulse shapes by oversampling and digital low-pass filtering. There is no design compromise between time resolution and complexity because the method gives continuous control over pulse shape and pulse duration.

While the new technique for computing excitation pulses is motivated by fitting excitation pulses to the inverse filter derived voice source, other applications include synthesizing low jitter speech waveforms for evaluating measures of voice aperiodicity (Milenkovic, 1987; Yumoto et al., 1982; Muta et al., 1988; Titze et al., 1987; Hillenbrand, 1987), the perceptual evaluation of low levels of jitter on voice quality (Hillenbrand, 1988), and the synthesis of the singing voice (Titze, 1986).

\section{METHODS}

We begin by deriving a formula for computing samples of a pulse waveform that has been low-pass filtered. We represent a pulse waveform which starts at $t=0$ and ends at $t=T$ as the piecewise polynomial function $p(t) u(t)$ $-p(t) u(t-T)$, where $u(t)$ is the unit step function and where the polynomial $p(t)$ has components

$$
p(t)=w_{1} p_{1}(\tau)+\cdots+w_{1 n} p_{1 n}(\tau),
$$

for $\tau=t / T$ and

$$
p_{i}(\tau)=p_{i 0}+p_{i 1} \tau+\cdots+p_{i n} \tau^{\prime \prime} .
$$

Synthesizer excitation waveform samples $p_{l_{d}}[n]$ for a pitch period beginning at $t_{d}$ will be obtained according to $p_{t_{d}}[n]=p\left(n t_{s}-t_{d}\right)$ where $t_{s}$ is the time between samples. The parameter $T$ controls pulse length while the parameters $w_{1}$ through $w_{m}$ control pulse shape.

The pulse waveform may be expressed as

$$
p(t) u(t)-p(t) u(t-T)=w P T_{n} u_{t}^{T}-w P B T_{n} u_{t-T}^{T},
$$

where $w$ is the row vector of pulse shape parameters $w_{i}, P$ is the matrix of polynomial weights $p_{i j}, T_{n}$ is a diagonal matrix of elements $1, T^{-1}, \ldots, T^{-n}, u_{t}$ is the vector $\left[u(t), t u(t), \ldots, t^{n} u(t)\right], \quad u_{t-r}$ is the vector $[u(t$ $\left.-T),(t-T) u(t-T), \ldots,(t-T)^{n} u(t-T)\right]$, and $B$ is a lower triangular matrix of binomial coefficients $b_{i j}$ coming from the expansion

$$
\begin{aligned}
\tau^{i} & =\{1+(\tau-1)\}^{i} \\
& =b_{i 0}+b_{i 1}(\tau-1)+\cdots+b_{i i}(\tau-1)^{n} .
\end{aligned}
$$

\section{A. Antialias filtering}

We compute samples of the pulse at discrete times $t=n t_{s}$. In order to suppress aliasing, we need to low-pass filter to a frequency at or below $1 /\left(2 t_{s}\right)$. This low-pass filter- 
ing needs to be performed in continuous time prior to computing sample values of the filtered pulse. To show how this is done, we note that the filtered pulse

$$
\begin{aligned}
p_{h}(t) & =h(t) *\{p(t) u(t)-p(t) u(t-T)\} \\
& =h(t) *\{p(t) u(t)\}-h(t) *[p(t) u(t-T)] \\
& =w P T_{n} h_{t}^{T}-w P B T_{n} h_{t-T}^{T},
\end{aligned}
$$

where * denotes convolution, $h_{t}$ is the vector

$$
\left[h(t) * u(t), h(t) * t u(t), \ldots, h(t) * t^{n} u(t)\right]
$$

and $h_{t-T}$ is the vector

$$
\begin{aligned}
& {[h(t) * u(t-T), h(t) *(t-T) u(t-T), \ldots,} \\
& \left.\quad h(t) *(t-T)^{n} u(t-T)\right] .
\end{aligned}
$$

Hence, filtering a piecewise polynomial pulse reduces to evaluating $h(t) * t^{i} u(t)$, where $h(t)$ is the filter impulse response. To evaluate $h(t) * t^{\prime} u(t)$, we define the linear operator

$$
\mathscr{L}\{g(t)\}=\int_{-\infty}^{t} g(u) d u,
$$

we note that

$$
\begin{aligned}
& u(t)=\mathscr{L}\{\delta(t)\}, \\
& t^{i} u(t)=t: \mathscr{L}^{i+1}\{\delta(t)\},
\end{aligned}
$$

and that

$$
h(t) * t^{\prime} u(t)=i ! \mathscr{L}^{i+1}\{h(t)\} .
$$

This last expression may be evaluated in closed form for any filter impulse response $h(t)$ that is $i$ times integrable.

The frequency sampling design (Gold and Jordan, 1969; Rabiner et al., 1970) gives a finite duration impulse response that is closed form integrable. The impulse response has the formula

$$
\begin{aligned}
h(t)= & h_{0}+h_{1} \cos \left(\pi t / t_{h}\right)+\cdots+h_{k} \cos \left(k \pi t / t_{h}\right), \\
& -t_{h}<t \leqslant t_{h},
\end{aligned}
$$

where impulse response $h(t)$ has duration $2 t_{h}$.

We evaluate $i ! \mathscr{L}^{i+1}\{h(t)\}$, essentially the normalized $i$ fold integration of $h(t)$, in the regions $t \leqslant-t_{h},-t_{h}<t \leqslant t_{h}$, and $t_{h}<t$. In first region, the expression evaluates to zero. In the second region, we will obtain a linear combination of sines and cosines as well as powers of $t$ through $t^{i+1}$. In the third region, $h(t)$ is zero valued, $\mathscr{L}\{h(t)\}$ will be a constant, and successive integrations will generate powers of $t$ through $t^{i}$. It follows that

$h_{\iota}=\left\{\begin{array}{l}0, \quad t \leqslant-t_{h} \\ C c_{t}^{T}+S s_{t}^{T}+U t_{n+1}^{T}, \quad-t_{h}<t \leqslant t_{h}, \\ V t_{n}^{T}, \quad t_{h}<t\end{array}\right.$

$h_{-T}=C c_{t-T}^{T}+S s_{t-T}^{T}+U d_{n+1}^{T}, \quad T-t_{h}<t \leqslant T+t_{h}$,

where we define the vectors

$$
\begin{aligned}
& c_{\imath}=\left[\cos \left(\pi t / t_{h}\right), \ldots, \cos \left(k \pi t / t_{h}\right)\right], \\
& s_{t}=\left[\sin \left(\pi t / t_{h}\right), \ldots, \sin \left(k \pi t / t_{h}\right)\right], \\
& t_{n+1}=\left[1, t, \ldots, t^{n+1}\right], \\
& t_{n}=\left[1, t, \ldots, t^{n}\right],
\end{aligned}
$$

$$
\begin{aligned}
& c_{t-T}=\left[\cos \left(\pi(t-T) / t_{h}\right), \ldots, \cos \left(k \pi(t-T) / t_{h}\right)\right], \\
& s_{t-T}=\left[\sin \left(\pi(t-T) / t_{h}\right), \ldots, \sin \left(k \pi(t-T) / t_{h}\right)\right], \\
& d_{n+1}=\left[1,(t-T), \ldots,(t-T)^{n+1}\right],
\end{aligned}
$$

and where $C, S, U$, and $V$ are matrices of constant coefficients.

The constant coefficient matrices may be computed by noting that rows $i$ of matrices $C, S, U$, and $V$ contain the coefficient weights of cosines, sines, and powers of $t$ making up $i \mathscr{L}^{i+1}\{h(t)\}$. That

$$
i ! \mathscr{L}^{i+1}\{h(t)\}=i \mathscr{L}\left\{(i-1) ! \mathscr{L}^{i}\{h(t)\}\right\}
$$

means row $i$ may be computed from row $i-1$ by following the rules for integrating sines, cosines, and powers of $t$

$$
\begin{aligned}
& \int \sin \left(\frac{j \pi t}{t_{h}}\right) d t=-\left(\frac{t_{h}}{j \pi}\right) \cos \left(\frac{j \pi t}{t_{h}}\right)+K, \\
& \int \cos \left(\frac{j \pi t}{t_{h}}\right) d t=\left(\frac{t_{h}}{j \pi}\right) \sin \left(\frac{j \pi t}{t_{h}}\right)+K, \\
& \int t^{j-1} d t=\left(\frac{1}{j}\right) t^{j}+K,
\end{aligned}
$$

where $K$ is the constant of integration in each expression. Matrix row $i=0$ is thus given by

$$
\begin{aligned}
& C_{0 j}=0 \quad 1 \leqslant j \leqslant k, \\
& S_{0 j}=\left(t_{h} / j \pi\right) h_{j} \quad 1 \leqslant j \leqslant k, \\
& U_{01}=h_{0}, \\
& U_{0 j}=0 \quad 1<j \leqslant n+1, \\
& V_{0 j}=0 \quad 1 \leqslant j \leqslant n,
\end{aligned}
$$

while rows $i=1$ through $i=n$ are given by

$$
\begin{aligned}
& C_{i j}=-\left(i t_{h} / j \pi\right) S_{i-1 j} \quad 1 \leqslant j \leqslant k, \\
& S_{i j}=\left(i t_{h} / j \pi\right) C_{i-1 j} \quad 1 \leqslant j \leqslant k, \\
& U_{i j}=(i / j) U_{i-1, j-1} \quad 1 \leqslant j \leqslant n+1, \\
& V_{i j}=(i / j) V_{i-1, j-1} \quad 1 \leqslant j \leqslant n .
\end{aligned}
$$

The values of the integration constants $K$ are obtained by forcing $h$, to be continuous at $t=-t_{h}$ and $t=t_{h}$. From Eq. (10), this condition is satisfied if $C c_{t}^{T}+S s_{t}^{T}+U t_{n+1}^{T}=0$ at $t=-t_{h}$ and if $C c_{1}^{T}+S s_{t}^{T}+U t_{n+1}^{T}=V t_{n}^{T}$ at $t=t_{h}$. It follows that for rows $i=0$ through $i=n$,

$$
\begin{aligned}
& -U_{i 0}=\sum_{j=1}^{n+1}\left(-t_{h}\right)^{i} U_{i j}+\sum_{j=1}^{k}(-1)^{j} C_{i j}, \\
& V_{i 0}=\sum_{j=0}^{n+1}\left(t_{h}\right)^{j}+\sum_{j=1}^{k}(-1)^{j} C_{i j}-\sum_{j=1}^{n+1}\left(t_{h}\right)^{j} V_{i j} .
\end{aligned}
$$

Combining Eqs. (5), (10), and (11), the filtered polynomial pulse reduces to

$$
p_{h}(t)=\left\{\begin{array}{rr}
c_{0} c_{1}^{T}+s_{0} s_{t}^{T}+u_{0} t_{n+1}^{T}, \quad-t_{h}<t \leqslant t_{h}, \\
v t_{n}^{T}, \quad t_{h}<t \leqslant T-t_{h}, \\
v t_{n}^{T}-\left(c_{1} c_{t-T}^{T}+s_{1} s_{t-T}^{T}+u_{1} d_{n+1}^{T}\right), \\
T-t_{h}<t \leqslant T+t_{h},
\end{array}\right.
$$

with coefficient vectors 
$c_{0}=w P T_{n} C, \quad s_{0}=w P T_{n} S, \quad u_{0}=w P T_{n} U, \quad v=w V$, $c_{1}=w P B T_{n} C, \quad s_{1}=w P B T_{n} S, \quad u_{1}=w P B T_{n} U$.

These coefficient vectors need to be computed for each pulse of new duration $T$ and shape parameters $w$.

Once $T$ and $w$ are fixed, the filtered polynomial pulse consists of two "fillets," the regions $-t_{h}<t \leqslant t_{h}$ and $T-t_{h}<t \leqslant T+t_{h}$, where the pulse is constructed from cosines, sines, and powers of $t$, together with the region $t_{h}<t \leqslant T-t_{h}$ where the pulse is simply a polynomial, of the same form as the original $p(t)$ but where the polynomial weights have been adjusted. The computational complexity compares favorable with, for example, simply "tacking on" fillet regions to the original polynomial pulse $p(t)$. The new technique, however, offers greater control over the spectral shape of the resulting pulse.

\section{B. Basis functions}

We control the shape of the piecewise polynomial pulse by adjusting the $w$ vector. The elements of the $w$ vector are weights for combining basis function polynomials $p_{i}(\tau)$ to obtain a representation of the first derivative of the glottal pulse. As we indicated earlier, we will be modeling an inverse filter signal that is the first derivative of the estimated glottal airflow.

The form of the basis functions is derived from a series of assumptions about the glottal pulse shape. We assume that the glottal flow starts at zero at the leading edge of the glottal pulse and ends at zero at the trailing edge. In the case where the glottis does not close completely on account of a glottal chink (Bless et al., 1986), we assume that glottal flow starts and ends at the same constant value. We will regard the glottal waveform to be a smooth function except at the points of glottal opening and closing, where the derivatives may be discontinous.

The dominant aspects of the excitation signal influencing the speech spectrum that we incorporate into our model are the pulse duration $T$ together with the discontinuities in the first two derivatives of the glottal wave at the glottal pulse end points. In the case of a glottal pulse which has a continuous first derivative between its end points, the asymptotic frequency spacing of the Laplace transform zeroes is controlled by the pulse duration $T$ while the asymptotic damping of these zeroes is controlled by the ratio of the closing edge to the opening edge slopes (Mathews et al., 1961; Flanagan, 1972). Our model will control the slope as well as the local curvature of glottal airflow at each pulse end point in order to better fit observed inverse filter waveforms.

The slope and curvature of the discontinuities of the glottal pulse are equivalent to the step and slope discontinuities of the glottal pulse derivative at its end points. We seek four basis functions of minimum polynomial order to exercise independent control over the step and slope discontinuities at the two end points. That the glottal pulse starts and ends at the same level imposes the constraint that the integrated value of each of the four basis functions be zero. The minimum polynomial order meeting these conditions is $n=4$ : each polynomial has five coefficients.
The four basis functions are polynomials $p_{1}(\tau)$ through $p_{4}(\tau)$ for $\tau=t / T$ where we specify boundary values

$$
p_{1}(0)=1, \quad p_{2}^{\prime}(0)=1, \quad p_{3}(1)=1, \quad p_{4}^{\prime}(1)=-1,
$$

where the remaining boundary values are set to zero. We add the contraint

$$
\int_{0}^{1} p_{i}(\tau) d \tau=0
$$

For each $p_{i}(\tau)$, evaluating Eq. (2) at the boundaries gives

$$
\begin{aligned}
& p_{i}(1)=p_{i 0}+p_{i 1}+p_{i 2}+p_{i 3}+p_{i 4}, \\
& p_{i}^{\prime}(1)=p_{i 1}+2 p_{i 2}+3 p_{i 3}+4 p_{i 4}, \\
& p_{i}(0)=p_{i 0}, \\
& p_{i}^{\prime}(0)=p_{i 1}, \\
& 0=p_{i 0}+\frac{1}{2} p_{i 1}+\frac{1}{3} p_{i 2}+\frac{1}{4} p_{i 3}+\frac{1}{5} p_{i 4} .
\end{aligned}
$$

Solving this linear system of equations for $i=1$ to $i=4$ results in

$$
P=\left[\begin{array}{ccccc}
1 & 0 & -18 & 32 & -15 \\
0 & 1 & -4.5 & 6 & -2.5 \\
0 & 0 & -12 & 28 & -15 \\
0 & 0 & -1.5 & 4 & -2.5
\end{array}\right]
$$

where $P$ is the matrix of elements $p_{i j}$, and

$$
P B=\left[\begin{array}{ccccc}
0 & 0 & -12 & -28 & -15 \\
0 & 0 & -1.5 & -4 & -2.5 \\
1 & 0 & -18 & -32 & -15 \\
0 & -1 & -4.5 & -6 & -2.5
\end{array}\right],
$$

where $B$ is the lower triangular matrix of binomial coefficients introduced in Eq. (4). Remembering that $P$ is the matrix of coefficients of the polynomials $p_{i}$ expressed as functions of $\tau$ and $P B$ is the matrix of coefficients of the same polynomials expressed as functions of $\tau-1$, the symmetry properties $\quad p_{1}(-\tau)=p_{3}(\tau-1) \quad$ and $\quad p_{2}(-\tau)$ $=p_{4}(\tau-1)$ account for the observed similarities between $P$ and $P B$.

Representative synthesizer pulses are depicted in Fig. 1; the Fourier transform of these pulses are seen in Fig. 2. The pulse weights are $w_{1}=50, w_{2}=0, w_{3}=-300$, and $w_{4}=1000$, and the sampling rate is $10 \mathrm{kHz}$. The pulses were produced under three conditions. The first condition is with no filter. The second condition is with frequency sampling filter $t_{h}=0.6 \mathrm{~ms}, h_{0}=0.5, h_{1}=1, h_{2}=1, h_{3}=1$, $h_{4}=0.3904$, which is a single transition sample low-pass design. The third condition is with the filter $t_{h}=0.6 \mathrm{~ms}$, $h_{0}=0.5, h_{1}=0.5$, which is a filter with a raised cosine impulse response. This third condition makes the smoothing effect of the filter more visually apparent.

Close examination of the glottal flow pulses in Fig. 1, obtained by integrating the sampled glottal flow derivative, reveals that the integrated pulse synthesized without the filter does not start and finish at the same level of closed glottis flow. The integrated pulses synthesized with an antialias filter do indeed start and finish at the same level. The antialias filter helps the sampled pulse of the glottal flow derivative to 

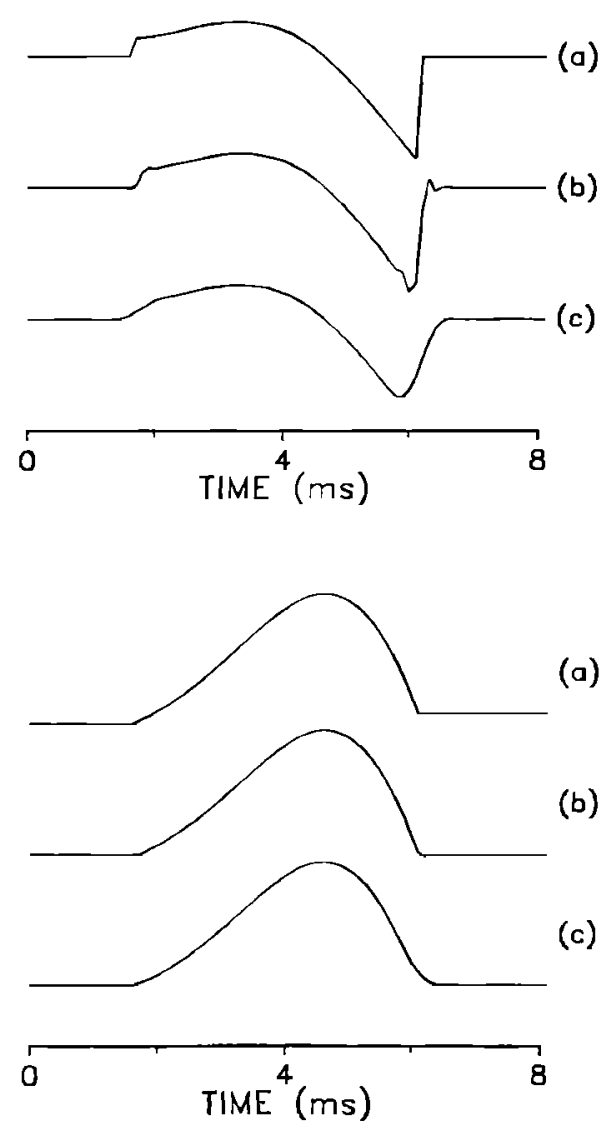

FIG. 1. Synthesized glottal pulses: (a) unfiltered, (b) frequency sampling design antialias filter, and (c) raised cosine filter. The top panel is glottal flow derivative, and the bottom panel is glottal flow.

maintain the same zero-dc constraint imposed on the continuous time version of the pulse.

\section{RESULTS}

We demonstrate three applications of the proposed voice source pulse model. The first is modeling the glottal wave by matching the pulse model to the inverse filtered acoustic waveform. The second is the use of the pulse model to synthesize waveforms used in the calibration of measures of voice perturbation. The third is the perceptual evaluation of low levels of voice perturbation in speech synthesis.

\section{A. Modeling the glottal wave}

An estimate of the glottal excitation waveform is obtained by LPC inverse filter analysis of the acoustic speech waveform. We recorded the acoustic wave with a Panasonic model WM-063T electret microphone cartridge (Digi-Key part P9932) AC coupled to a preamplifier with a high-pass cutoff frequency of $2 \mathrm{~Hz}$. The microphone is held in close proximity to the subject's lips $(5 \mathrm{~cm})$ by a boom attached to a pair of safety glasses worn by the subject. The microphone is held off-axis to avoid air-blast artifact. Placing the microphone in the near field maximizes the ratio of direct sound energy to the energy of sound reflected from the room, minimizing artifact resulting from reverberation.

The output of the microphone preamplifier was lowpass filtered at $4 \mathrm{kHz}$ with a four-pole Butterworth filter and

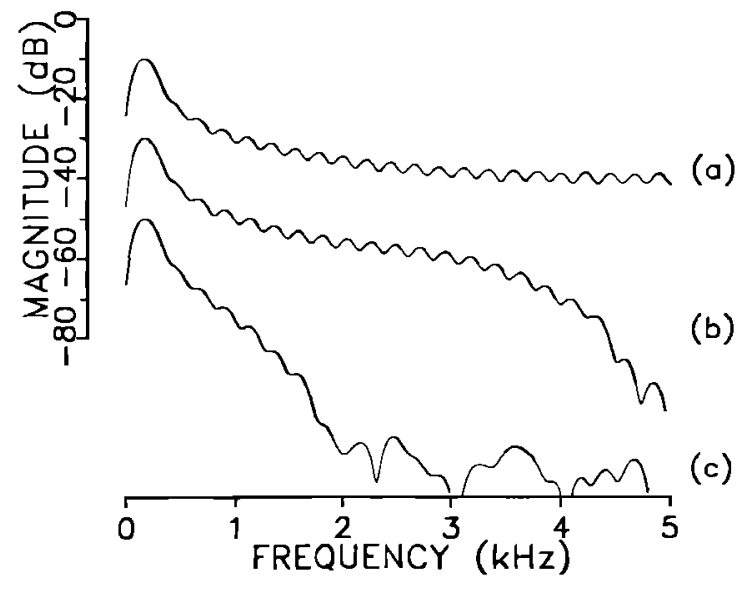

FIG. 2. Fourier magnitude spectrum of the glottal flow derivative for synthesized pulses. Synthexis conditions: (a) unfiltered, (b) frequency sampling design antialias filter, and (c) raised cosine filter.

sampled at $8.33 \mathrm{kHz}$ using an A/D with 12 bits resolution. The digital speech recording is high pass filtered by two applications of a single pole high-pass digital filter with a $20-\mathrm{Hz}$ cutoff. The high-pass filter is first applied to the data in the forward direction and then is applied to the data in the backward direction, resulting in a two-pole filter with zero phase (Milenkovic, 1987). Use of a zero-phase high-pass filter minimizes the waveform droop, making the distinction between the open and closed phases of the glottal pulse more apparent.

The inverse filter is determined by applying ten coefficient covariance method LPC analysis to the speech wave, preemphasized by taking the first difference (Markel and Gray, 1976). This analysis is applied to a 20-ms interval of the waveform. Applying the resulting LPC inverse filter to the unpreemphasized speech waveform recorded with a pressure sensing microphone gives an estimate of the first derivative of the glottal airflow (Milenkovic, 1987). Integrating the inverse filter signal gives an estimate of the ac component of glottal flow.

We collected data from four male and four female subjects in order to obtain representative waveforms. We instructed our subjects to sustain the vowel /a/ for $1 \mathrm{~s}$. We employed the vowel /a/ because the high first formant frequency of this vowel reduces the artifact caused by misalignment of the inverse filter zeros with the vocal tract poles. Figure 3 shows both Fourier as well as LPC spectra of the preemphasized speech signals for one male and two female subjects. We show one female subject having a spectrogram similar to the male subject. We show another female subject with a spectrogram that is most dissimilar to the male subject, exhibiting the elevated first harmonic together with broadened formant bandwidths described as typifying female vowels (Klatt, 1980; Klatt and Klatt, 1990).

A computer program identified successive pitch periods by comparing the large downward stroke of the inverse filter signal against a threshold. The downward stroke identifies the approximate location of glottal closure. The computer program then fit a five-parameter pulse model to the inverse filter waveform in each pitch period. The five parameters are 


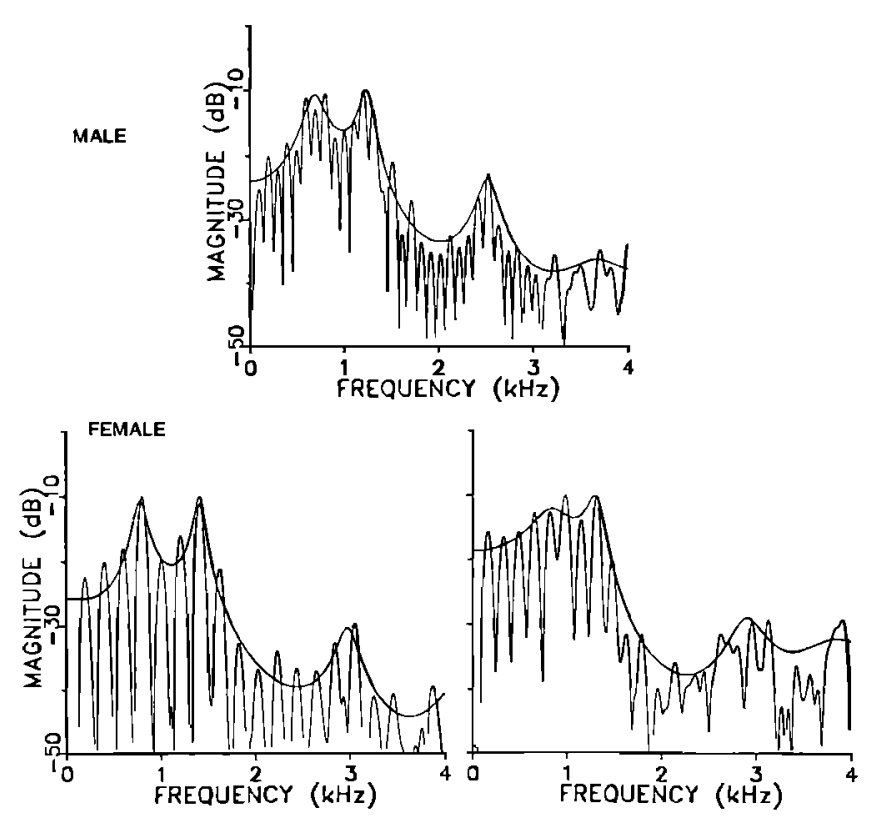

FIG. 3. Fourier and LPC spectra of the vowel /a/.

$t_{1}$ and $t_{2}$, the times of glottal opening and glottal closing, as well as $w_{1}, w_{3}$, and $w_{4}$, the weights of basis polynomials $p_{1}$, $p_{3}$, and $p_{4}$. We excluded $p_{2}$ by fixing $w_{2}$ at zero as we were able to obtain good fits without this basis function.

In fitting the pulse model to the inverse filter waveform, we employed a raised cosine filter with parameters $t_{h}=0.24$ $\mathrm{ms}, h_{0}=0.5, h_{1}=0.5$. This filter deemphasizes the higher frequencies and has been empirically selected for its effectiveness in the pulse fitting process. One may readily change

MALE

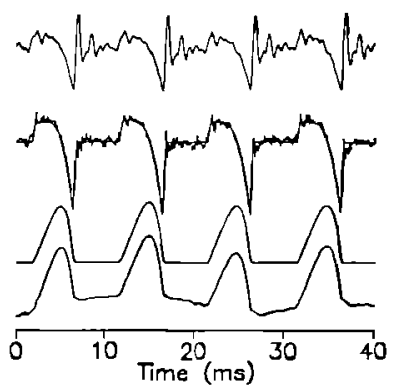

FEMALE
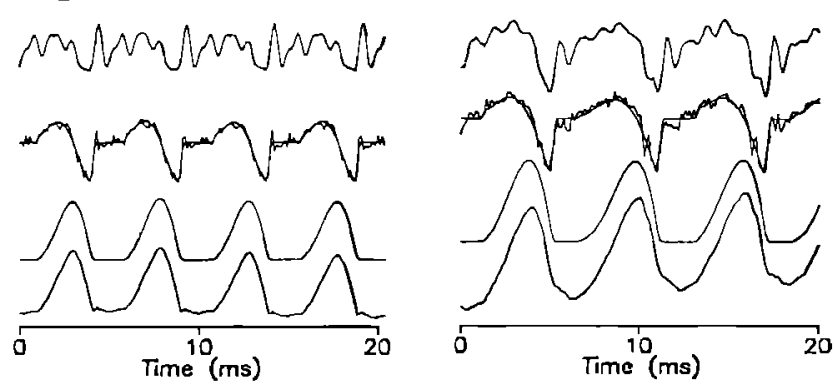

FIG. 4. Voice source waveforms: upper: free-field acoustic pressure, upper middle: superimposed pulse model and inverse filter estimate of the first derivative of glottal airflow, lower middle: integrated pulse model, and lower: inverse filter estimate of glottal flow. the $h$ coefficients to substitute a broader band filter for later speech synthesis using the fitted glottal pulses.

The parameters $t_{1}$ and $t_{2}$ may be adjusted over continous time because of the antialias property of the method for computing pulse samples. For each trial value of $t_{1}$ and of $t_{2}$, we obtain values of $w_{1}, w_{3}$, and $w_{4}$ by linear regression of the inverse filter waveform against the scaled, filtered, and time shifted basis polynomials $p_{1}, p_{3}$, and $p_{4}$. We first step through values of $t_{2}$ with $t_{1}$ held fixed, then step through values of $t_{1}$ with $t_{2}$ held fixed in order to find values of $t_{1}$ and $t_{2}$ that give the best least-squares regression on the linear weights $w_{1}, w_{3}$, and $w_{4}$. We repeat this process, each time reducing the step size used to adjust $t_{1}$ and $t_{2}$, until we obtain optimal values for all five parameters.

The results of fitting the pulse model are shown in Fig. 4. With the second female subject, we observed cycle-to-cycle fluctuation in the value of $t_{1}$, the glottal opening time. This is not unique to females; we show results for a male subject exhibiting this condition in Fig. 5. This figure includes a contour plot, indicating contour lines of equal least-squares regression on the $w$ 's for different values of the end points $t_{1}$ and $t_{2}$. The contour plot was obtained by sampling $t_{1}$ and $t_{2}$ on a grid ten times denser than the 0.12 -ms sample spacing and by employing the Surfer software package (Golden Software, Golden, Colorado). This contour plot indicates that there are multiple local minima in $t_{1}$, and these minima are of nearly the same magnitude. This suggests that the precise moment of glottal opening is ambiguous given the degrees of freedom in our model and the degree to which the
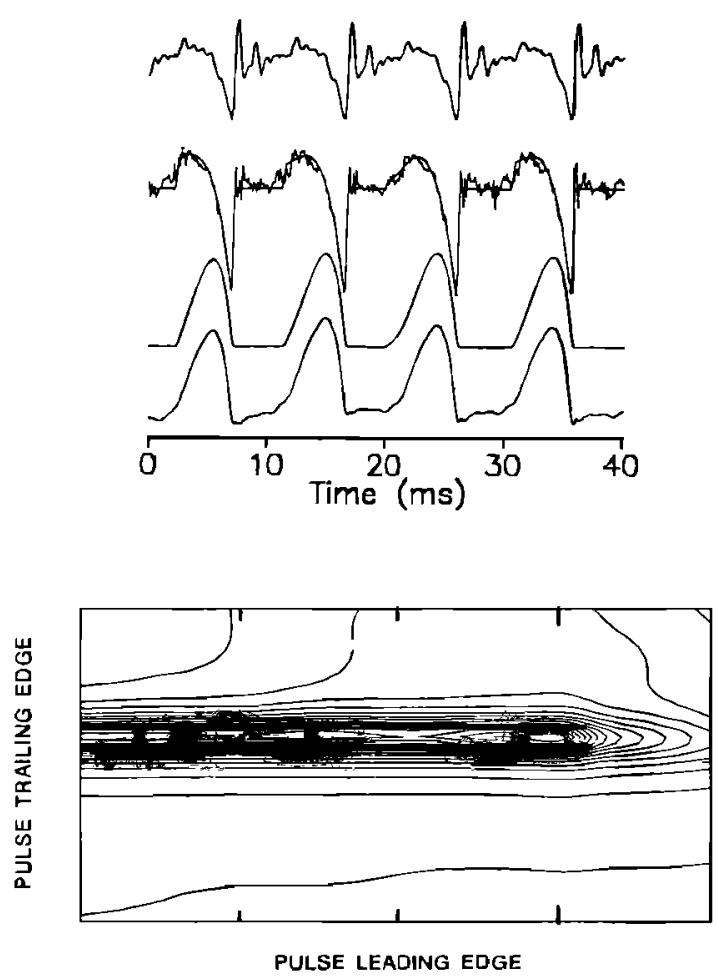

FIG. 5. Male subject exhibiting ambiguity in the time of glottal opening. The bottom panel is a contour plot of the least-squares fit between the pulse model and the glottal flow derivative as a function of the pulse end points. The tick marks are placed one sample point $(0.12 \mathrm{~ms})$ apart. 
model fits inverse filter waveforms. This ambiguity is inherent in the fit between the model and the data and does not appear to be an artifact of the search procedure used to find optimal values of $t_{1}$ and $t_{2}$.

\section{B. Calibration of voice perturbation}

We used the voice source pulse model to synthesize constant pitch synthetic vowels for purposes of calibrating measures of jitter and shimmer. The naturally occurring jitter may be as low as $10 \mu \mathrm{s}$ while shimmer may be under $1 \%$ (Milenkovic, 1987). Ideally, one would like a synthetic voice source where jitter and shimmer may be controlled to well below these levels in order to determine the noise floor on the algorithms used to measure jitter and shimmer. This synthetic voice source should be capable of producing pitch periods that are not restricted to an integer number of sample points because the natural voice source does not have this restriction.

We employed the same pulse shape and $h(t)$ filter depicted in Fig. 1. The pulse weights were set at $w_{1}=50$, $w_{2}=0, w_{3}=-300$, and $w_{4}=1000$, we fixed the open portion of the glottal pulse at 0.55 the pitch period, and we selected a sampling rate of $10 \mathrm{kHz}$. The antialias filter was given the specifications $t_{h}=0.6 \mathrm{~ms}, h_{0}=0.5, h_{1}=1$, $h_{2}=1, h_{3}=1, h_{4}=0.3904$, which is a single transition sample low pass design. The voice source waveform produced in this manner was fed into a 12 coefficient LPC synthesizer. The LPC coefficients were obtained by covariance method analysis applied to a 20-ms duration of the preemphasized speech waveform, applied to the vowel /a/ produced by a male subject.

Three synthesis conditions were used. The first condition is synthesis without the use of the $h(t)$ filter where the pulse end points were rounded to the nearest sample point; this is the pitch synchronous condition. The pitch periods were then adjusted to maintain the average pitch period value. The second condition is synthesis without the $h(t)$ filter where the voice source pulse is computed asynchronously for a pitch period that is not an integer number of sample points. The voice source pulse is treated as a continuous time waveform which is sampled without regard for the resulting aliasing. In the third condition, the voice source pulse is computed asynchronously, but this time with the benefit of the antialias filter $h(t)$.
Synthesis pitch periods were selected from two ranges. The range around $8 \mathrm{~ms}$ is representative of a male voice while the range around $4 \mathrm{~ms}$ is representative of a female voice. For each range, pitch period values were selected that were noninteger numbers of sample points. The values 8.05 and 4.05 $\mathrm{ms}$ are midway between integer multiples of the sampling interval, and we expect synthesis jitter to be greatest at these values.

The values of jitter and shimmer are obtained from a 250-ms interval of a synthesized vowel of constant pitch; the analysis was performed according to a method described by Milenkovic (1987). Results are summarized in Table I. In this analysis procedure, jitter and shimmer are obtained by computing the short-term autocorrelation function and using parabolic interpolation to find the peak. The interpolated peak of the short-term autocorrelation function provides an accurate measure of pitch period from which to compute jitter, and the amplitude of this peak is used to compute shimmer.

In the synthesis conditions without an $h(t)$ filter, we observe considerable shimmer, the value of which varies with the pitch range as well as the synthesis method. In the pitch synchronous synthesis without the $h(t)$ filter, shimmer is attributable to the jittered overlap of the vocal tract impulse response between successive pitch periods. Considerable reduction in both jitter and shimmer is obtained by using the $h(t)$ filter to compute the voice source pulses.

\section{Perceptual effect of aliasing artifact}

The question arises whether the reduction in jitter and shimmer afforded by the new source model is perceptually significant. We present results of listening tests on the ability of subjects to detect differences between vowel tokens synthesized with and without the antialiasing filter. These tests constitute a demonstration that the proposed voice source model has perceptual relevance.

There are two ways to synthesize the voice source pulses without the benefit of the antialias filter. In the pitch synchronous case, we move the pulse end points to the nearest sample points; in the pitch asynchronous case, we place the pulse end points between sample points and sample with aliasing. Looking at Table I, both the synchronous and asynchronous cases resulted in nearly the same level of jitter. The asynchronous case, however, has much higher values of shimmer at the low-constant pitch setting. On the other

TABLE I. Measured jitter and shimmer for the synthesized vowel /a/ sampled at $10 \mathrm{kHz}$.

\begin{tabular}{|c|c|c|c|c|c|c|}
\hline \multirow[b]{3}{*}{$\begin{array}{l}\text { Pitch period } \\
\text { (ms) }\end{array}$} & \multirow{2}{*}{\multicolumn{2}{|c|}{$\begin{array}{c}\text { Pitch synchronous } \\
\text { No filter }\end{array}$}} & \multicolumn{4}{|c|}{ Pitch asynchronous } \\
\hline & & & \multicolumn{2}{|c|}{ No filter } & \multicolumn{2}{|c|}{ Low-pass filter } \\
\hline & $\begin{array}{l}\text { jitter } \\
(\mu \mathrm{s})\end{array}$ & $\begin{array}{c}\text { shimmer } \\
(\%)\end{array}$ & $\begin{array}{l}\text { jitter } \\
(\mu s)\end{array}$ & $\begin{array}{l}\text { shimmer } \\
(\%)\end{array}$ & $\begin{array}{l}\text { jitter } \\
(\mu \mathrm{s})\end{array}$ & $\begin{array}{c}\text { shimmer } \\
(\%)\end{array}$ \\
\hline 8.02 & 35 & 0.31 & 34 & 2.27 & 0 & 0.13 \\
\hline 8.05 & 70 & 0.60 & 69 & 3.23 & 0 & 0.09 \\
\hline 4.02 & 42 & 3.28 & 40 & 3.61 & 1 & 0.15 \\
\hline 4.05 & 87 & 8.23 & 86 & 3.46 & 1 & 0.18 \\
\hline
\end{tabular}


hand, the pitch synchronous case has higher shimmer at the high-constant pitch setting. We attribute this effect to the overlap of impulse responses between successive pitch periods and contructive and destructive interference as the pulses are jittered.

We limited the comparison to two conditions to simplify the perceptual task for our subjects. Given the importance of demonstrating the perceptual differences at low pitch values, where the percent jitter is much smaller, we compared these two synthesis conditions. The first condition was pitch asynchronous with the antialias filter. The second condition was pitch synchronous without the antialias filter. In this second synthesis condition, we followed the pulse synthesis with the application of a digital filter to match the spectrum of the first synthesis condition.

The two synthesis conditions have closely matched spectra as seen in Fig. 6, eliminating spectrum envelope difference as a perceptual clue. The two spectra, however, differ in the amount the harmonic peaks are separated from the apparent noise floor; this difference is inherent in the two synthesis methods as this spectrographic effect is not readily compensated by post filtering. We seek to determine if such spectrum differences are perceptually apparent.

The good match between spectrum envelopes is a consequence of the pair of conditions selected for comparison. Under the pitch synchronous condition, the waveshape of the sampled voice source pulse stays exactly the same from cycle to cycle-only the pitch period is changed. This means that apart from changes in the spectrum fine structure, the two synthesis methods give virtually the same spectrum. In the case of pitch asynchronous synthesis without the antialias filter, the wave shape of the pulse after sampling can vary with pulse alignment with the sampling points. By not including this case, we eliminate small changes in spectrum shape as a factor in any perceptual differences that occur.

We synthesized 500-ms tokens of the vowel /a/ sampled at $10 \mathrm{kHz}$ according to the procedure of the previous section. We applied an exponential taper to the amplitude envelope of both the onset and offset of each vowel token. The pulse amplitudes at onset were given by $A_{0}\left(1-0.5^{n}\right)$, where $A_{0}$ is the steady-state pulse amplitude and $n$ is the pitch period number after onset. The pulse amplitudes at offset were given by $A_{0}(0.8)^{n}$, where $n$ is the pitch period number after offset.

We synthesized vowel tokens with three different pitch inflections. The first was a constant low pitch with a pitch period of $8.05 \mathrm{~ms}$. The second was a falling high pitch with an initial pitch period of $4 \mathrm{~ms}$ and a final pitch period of 4.4 ms. The third was a falling low pitch with an initial pitch period of $8 \mathrm{~ms}$ and a final pitch period of $8.8 \mathrm{~ms}$.

We first conducted informal listening evaluation of these tokens. The informal evaluation indicated that the first condition, with a true constant pitch period, gave a less rough sound quality than the second condition, with a constant average pitch period maintained by aligning pulse end points at the nearest sample points. The three tokens, as we have ordered them, turn out to be ranked in order of most apparent to least apparent for this effect.

The differences between synthesis conditions were ap-
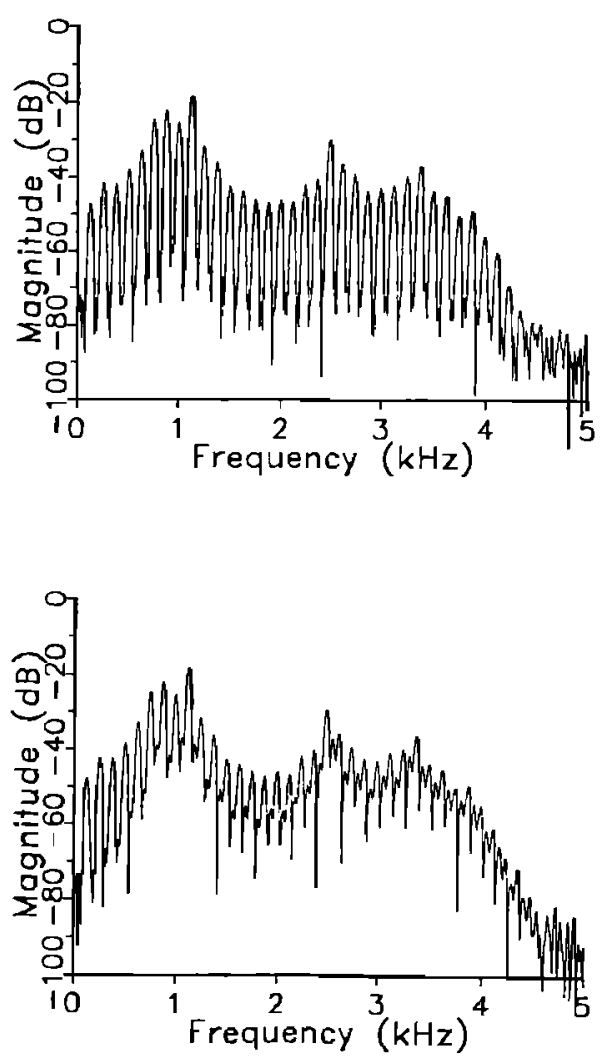

FIG. 6. Fourier analysis of the synthetic vowel $/ a /$. Use of the antialias filter to synthesize a vowel with a pitch period that is a noninteger multiple of the sample interval is shown at the top. Rounding the glottal pulse end points to the nearest sample position and then post filtering to match the spectrum envelope is shown at the bottom.

parent when the tokens were output over a loudspeaker. The differences vanished when the tokens were output over headphones. Hillenbrand (1988) reported the same effect in listening evaluation of low levels of voice perturbation. Wilde et al. (1986) indicated that the roughness of jittered sounds is most apparent in a reverberant sound field or in sound presented over headphones with artificial reverberation. On the basis of these results, we decided to conduct our formal listening evaluation free-field over a loudspeaker.

We employed six male subjects, ages 18 to 30 years, for our formal listening trials. We presented pairs of vowel tokens over a loudspeaker placed $6 \mathrm{ft}$ from the subject. The listening was performed in a room typical of an office environment. The pairs of tokens were random selections from the set of ordered pairs $\{A A, A B, B A, B B\}$, where $A$ denotes the first synthesis condition and $B$ denotes the second. The two 500 -ms tokens were separated by $200 \mathrm{~ms}$. After each presentation, the subject indicated "same," "different," or "unable to decide" by the appropriate key press on a computer keyboard. The next token pair was presented as soon as the subject indicated a response for the previous pair.

The subjects were instructed to listen for a smooth/ rough distinction in the vowel tokens. They were not given any prior training in terms of practice trials. Each subject was presented with 100 trials of each of the three vowel tokens, starting with constant low pitch and ending with falling low pitch. The results are summarized in Table II. A result of 66 or more correct answers is significant at $p<0.001$ 
TABLE II. Discriminations between aliased voice source and antialias filtered voice source made by six subjects. $C$ : correct; $I$ : incorrect; $A$ : abstain.

\begin{tabular}{cccccccccc}
\hline \hline & \multicolumn{4}{c}{ Constant low pitch } & \multicolumn{3}{c}{ Falling high pitch } & \multicolumn{3}{c}{ Falling low pitch } \\
Subject & C & I & A & C & I & A & C & I & A \\
& & & & & & & & & \\
\hline 1 & 96 & 2 & 2 & 97 & 1 & 2 & 75 & 18 & 7 \\
2 & 94 & 4 & 2 & 95 & 2 & 3 & 80 & 14 & 6 \\
3 & 93 & 6 & 1 & 96 & 4 & 0 & 74 & 26 & 0 \\
4 & 82 & 13 & 5 & 98 & 1 & 1 & 58 & 40 & 2 \\
5 & 98 & 1 & 1 & 99 & 0 & 1 & 86 & 8 & 6 \\
6 & 98 & 2 & 0 & 64 & 36 & 0 & 43 & 50 & 7 \\
\hline \hline
\end{tabular}

for the binomial distribution. The first token was correctly discriminated by all six subjects, the second by five subjects, and the third by four subjects. The subject who failed to discriminate the second token also failed to discriminate the third token, consistent with our ordering of the difficulty of the tasks.

\section{IIt. DISCUSSION}

The proposed scheme for synthesizing voice source pulses with end points controllable between sample times offers definite advantages when fitting pulses to the inverse filter waveform. It permits us to treat the glottal opening and closing times as real instead of integer parameters in optimization algorithms for determining the glottal opening and closing times that produce an optimal fit. The more precise specification of the pulse end points can improve the fit of the source model to the inverse filter waveform. It also aids in mapping the least-square error surface as a function of the pulse end-point locations, giving insight into the location of local minima and the effect of these minima on an optimization algorithm.

The map of the least-squares error as a function of pulse end points indicates an inherent ambiguity in the glottal opening transient. The glottal closing transient is marked by a strong global optimum of the error. The glottal closing transient, however, can be marked by multiple local minima of nearly the same least-square error. This ambiguity of the glottal opening transient suggests that the pulse model has more degrees of freedom than can be resolved from one pitch period cycle rather than the fault being with the optimization algorithm. Imposing a smooth variation in the glottal opening time between pitch period cycles might be a way to resolve this ambiguity.

We were able to fit inverse filter waveforms of both male and female subjects with our pulse model, even though we have not encorporated the exponential tail following the glottal closing transition, a feature of the LF model (Fant and Lin, 1988). The effect of this exponential tail is to change the spectral tilt of the speech signal. Klatt and Klatt (1990) propose to add the spectral tilt by filtering a source pulse without the exponential tail; the filtered pulsc will exhibit such a tail. In our case, where covariance LPC analysis is applied to an analysis frame containing at least one pitch period, this spectral tilt is incorporated into the vocal tract filter instead of the source pulse.

The acoustic speech waveform is readily resynthesized by exciting an LPC vocal tract filter with the sequence of pulses extracted from the inverse filter waveform. The voice source pulses can have a bandwidth close to the Nyquist bandwidth for the sample rate used. The perceptually significant jitter and shimmer that results from aligning the pulse end points at discrete sample positions is eliminated.

In synthesis applications where one is not required to align each pitch period with the pitch period in a natural utterance, the pitch period could be rounded to the nearest integer number of sample intervals. While perceptual tests with constant pitch sounds suggest a pitch discrimination threshold of $0.3 \mathrm{~Hz}$ (Flanagan and Saslow, 1958), experiments on sounds with varying pitch indicate a threshold of 2 $\mathrm{Hz}$ (Klatt, 1973), and experiments with sentences indicate a threshold of $4 \mathrm{~Hz}$ (Mack and Gold, 1986).

These studies of pitch discrimination, however, address the issue of pitch shifts between pairs of syllable or sentence length utterances. Pitch fluctuations between successive pitch periods, known as jitter, is readily perceptable as roughness at the level of $1 \%$ (Hillenbrand, 1988) ( $1 \mathrm{~Hz}$ for a pitch of $100 \mathrm{~Hz}$ ). The jitter levels of natural speech have average values on the order of $0.5 \%$ (Horii, 1979) and have been measured as low as $0.1 \%$ (Milenkovic, 1987). Fine control of the pitch period is required to produce jitter at or below these levels.

While Klatt and Klatt (1990) argue against the low levels of jitter in natural speech being perceptually significant in sentence productions, they incorporate a pitch "flutter" into their synthesizer, a sinusoidal variation in pitch over a long time interval. The accurate synthesis of flutter would benefit from our proposed technique. It can be readily demonstrated that slow pitch changes in the Klatt synthesizer (Klatt, 1980) can be heard as a series of steps, and our technique can eliminate this effect. The fine control of pitch is also important in the synthesis of the singing voice to eliminate beats when combining synthesized singing with other musical sounds.

The ability to exercise continuous control of pitch is an important tool in both the perceptual as well as algorithmic measurement of voice perturbation. One no longer needs to make a judgment as to what level of oversampling is adequate in perceptual experiments of pitch and of jitter and shimmer. The proposed synthesis method can also provide an accurate standard for comparing different methods of measuring jitter and shimmer.

\section{ACKNOWLEDGMENT}

Work was supported by NIDCD Grant No. P60 DC00976.

Ananthapadmanabha, T. V., and Fant. G. (1982). "Calculation of irue glottal flow and its components," in Speech Communication (North Holland, Amsterdam), Vol. 1, pp. 167-184.

Atal, B. S., Chang, J. J., Mathews, M. V., and Tukey. J. W. (1978). "Inversion of articulatory-to-acoustic transformation in the vocal tract by a computer-sorting technique," J. Acoust. Soc. Am. 63, 1535-1555.

Atal, B. S., and Remde, J. R. (1982). "A new model of LPC excitation for producing natural-sounding speech at low bit rates," IEEE ICASSP Proc. 614-617. 
Bless, D. M., Biever, D., and Shaikh, A. ( 1986). “Comparisons of vibratory characteristics of young adult males and females," in Proceedings of the International Conference on Voice, Kurume, Japan, Vol. 2, pp. 46-54.

Childers, D. G., and Lee, C. K. (1991). "Vocal quality factors: Analysis, synthesis, and perception," J. Acoust. Soc. Am. 90, 2394-2410.

Fant, G., and Lin, Q.-G. (1988). "Frequency domain interpretation and derivation of glottal flow parameters," STL-QPSR 2-3, 1-21.

Flanagan, J. L. (1972). Speech Analysis, Synthesis and Perception (Springer-Verlag, Berlin ), 2nd ed.

Flanagan, J. L., and Saslow, M. G. (1958). "Pitch discrimination in synthetic vowels," J. Acoust. Soc. Am. 30, 435-442.

Gold, B., and Jordan, K. L. (1969). “A direct search procedure for designing finite duration impulse response filters," IEEE Trans. Audio Electroacoust. AU-17, 33-36.

Hartwell, W. T., and Prezas, D. P. (1981). "A pulse driving function generator for LPC synthesis of voiced segments of speech," IEEE Trans. Acoust. Speech Signal Proc. ASSP-29, 1113-1116.

Hillenbrand, J. (1987) . "A methodological study of perturbation and additive noise in synthetically generated voice signals," J. Speech Hear. Res. 30, 448-461.

Hillenbrand, J. (1988). "Perception of aperiodicities in synthetically generated voices," J. Acoust. Soc. Am. 83, 2361-2371.

Holmes, J. N. (1973). "The influence of glottal waveform on the naturalness of speech from a parallel formant synthesizer," IEEE Trans. Audio Electroacoust. AU-21, 298-305.

Horii, Y. (1979). "Fundamental frequency perturbation observed in sustained phonation," J. Speech Hear. Res. 22, 5-19.

Klatt, D. H. (1973). "Discrimination of fundamental frequency contours in synthetic speech-implications for models of pitch perception," $J$. Acoust. Soc. Am. 53, 8-16.

Klatt, D. H. (1980). "Software for a cascade/parallel formant synthesizer," J. Acoust. Soc. Am. 67, 97I-995.

Klatt, D. H., and Klatt, L. C. (1990). "Analysis, synthesis, and perception of voice quality variations among female and male talkers," J. Acoust. Soc. Am. 87, 820-857.

Mack, M., and Gold, B. (1986). "The effect of linguistic content upon the discrimination of pitch in monotone stimuli," J. Phon. 14, 333-337.

Markel, J. D., and Gray, A. H., Jr. (1976). Linear Prediction of Speech (Springer-Verlag, Berlin).
Mathews, M. V., Miller, J. E., and David, E. E., Jr. (1961). "Pitch synchronous analysis of voiced sounds," J. Acoust. Soc. Am. 33, 179-186.

Milenkovic, P. (1984). "Vocal tract area functions from two-point acoustic measurements with formant frequency constraints," IEEE Trans. Acoust. Speech Signal Proc. ASSP-32, 1122-1 135.

Milenkovic, P. (1986). "Glottal inverse filtering by joint estimation of an $A R$ system with a linear input model," IEEE Trans. Acoust. Speech Signal Proc. ASSP-23, 28-42.

Milenkovic, P. (1987). "Least mean square measures of voice perturbation," J. Speech Hear. Res. 30, 529-538.

Muta, H., Baer, T., Wagatsuma, K., Muraoka, T., and Fukuda, H. (1988). "A pitch-synchronous analysis of hoarseness in running speech," $\mathrm{J}$. Acoust. Soc. Am. 84, 1292-1301.

Pinto, N. B., Childers, D. G., and Lalwani, A. L. (1989). "Formant speech synthesis: improving production quality," IEEE Trans. Acoust. Speech Signal Process. ASSP-37, 1870-1887.

Rabiner, L. R., Gold, B., and McGonegal, C. A. (1970). "An approach to the approximation problem for nonrecursive digital filters," IEEE Trans. Audio Electroacoust. AU-18, 83-106.

Rosenberg, A. E. (1971). "Effect of glottal pulse shape of the quality of natural vowels," J. Acoust. Soc. Am. 49, 583-590.

Rothenberg, M. (1983). "An interactive model for the voice source," in Vocal Fold Physiology, edited by D. M. Bless and J. H. Abbs (College Hill, San Diego).

Sondhi, M. M., and Schroeter, J. (1988). "A hybrid time-frequency domain articulatory synthesizer," IEEE Trans. Acoust. Speech Signal Proc. ASSP-35, 955-967.

Titze, I. R. (1986). "Three models of phonation," J. Acoust. Soc. Am. Suppl. 1 79, S81-S82.

Titze, I. R., Horii, Y., and Scherer, R. C. ( 1987). "Some technical considerations in voice perturbation measurements." J. Speech Hear. Res. 30, 252-260.

Wilde, M. D., Martens, W. L., Hillenbrand, J., and Jones, D. R. (1986). "Externalization mediates changes in the perceived roughness of sound signals with jittered fundamental frequencies," in Proceedings of the 1986 Intemational Computer Music Conference, The Hague.

Yumoto, E., Gould, W. J., and Baer, T. (1982). "Harmonics-to-noise ratio as an index of the degree of hoarseness," J. Acoust. Soc. Am. 71, 1544 1550. 